\title{
Calcification of valved aortic allografts in rats: Effects of age, crosslinking, and inhibitors
}

\author{
Robert J. Levy, ${ }^{1}$ Xuan Qu, ${ }^{1}$ Thomas Underwood, ${ }^{1}$ Joseph Trachy, ${ }^{1}$ and Frederick J. Schoen ${ }^{2}$ \\ ${ }^{1}$ University of Michigan Medical School, Ann Arbor, Michigan; ${ }^{2}$ Brigham and Women's Hospital, Boston, \\ Massachusetts
}

\begin{abstract}
Experiments were carried out to investigate rat aortic allograft calcification using valved abdominal aortic allografts. Results indicated that this was a potentially useful model for investigating fresh allograft calcification, as well as mineralization of glutaraldehyde-crosslinked valved allografts. Valve cusp results, however, were not comparable to those noted in large animal or human studies, while aortic wall calcification was more comparable. Calcification inhibitor investigations demonstrated that nearly complete inhibition of the calcification of the aortic wall of glutaraldehyde-crosslinked allografts was achieved using a number
\end{abstract}

of individual inhibitors, including controlled release diphosphonates, and pretreatment with either ferric chloride or aluminum chloride. However, aminopropanehydroxydiphosphonate pretreatment was not efficacious, and sodium dodecyl sulfate pretreatment was only partially effective for inhibiting the aortic wall calcification in the glutaraldehyde-crosslinked allografts. It is concluded that valved aortic allografts in rats provide a useful model for investigating aortic wall (but not valve cusp) calcification and its inhibition. (C) 1995 John Wiley \& Sons, Inc.

\section{INTRODUCTION}

Cardiac valve replacement surgery is frequently carried out utilizing tissue derived valvular prostheses consisting of either human allograft ${ }^{1-5}$ or xenograft bioprosthetic valves. ${ }^{6-13}$ Xenograft valve prostheses have most commonly been constructed from glutaraldehyde-crosslinked porcine aortic valves ${ }^{6-10}$ or bovine pericardium, ${ }^{11-13}$ utilized as stentmounted bioprostheses. Autografts are most often used as unfixed stentless valved conduits, but glutaraldehyde-fixed porcine aortic valves have also been recently investigated. ${ }^{14-18}$ Clinical failure due to cuspal calcification is a problem common to both allograft ${ }^{1-5}$ and bioprosthetic replacement valves. ${ }^{6-13}$ In addition, aortic wall calcification is an important problem that limits the longevity of aortic allografts ${ }^{19,20}$ and is likely to affect stentless bioprostheses. $^{21}$

Previous animal models of bioprosthetic and allograft valve calcification degeneration have utilized either subdermal implants in small animals, such as rats, ${ }^{22-28}$ or cardiac valve implants (orthotopic or ven-

*To whom correspondence should be addressed at University of Michigan, Department of Pediatrics, R5014 Kresge II 0576 Ann Arbor, MI 48109. tricular-conduit) using sheep ${ }^{29-32}$ or calves. ${ }^{33,34}$ These animal model systems have provided important insights into the pathogenesis of the calcific degeneration of tissue-derived cardiac valve prostheses, and they have been used to investigate preventive therapeutic approaches. Glutaraldehyde-crosslinked bioprostheses calcify due to an interaction of host and implant factors. ${ }^{35}$ The most important host factor has been shown to be the calcium metabolism of the recipient, best exemplified by the potentiating effect of young age of the implanted subject. ${ }^{7,22}$ Immature recipients, either animals or human subjects, implanted with glutaraldehyde-crosslinked porcine aortic valve cusps, experience more rapid implant calcification than mature subjects. ${ }^{7,22}$ The most important implant factor has been shown to be glutaraldehyde crosslinking. ${ }^{22}$ Rat subdermal implants studies of porcine aortic cusps demonstrated that over a 3-week time course, glutaraldehyde-crosslinked porcine aortic cusps developed calcification intrinsic to the cuspal structure, that did not occur in fresh, noncrosslinked implants. ${ }^{22}$ Moreover, the calcification loci were intimately related to the tissue constituents, particularly the original connective tissue cells that were transplanted as part of donor valve. ${ }^{22,24}$

Calcification of fresh or cryopreserved valved allografts is less well understood. However, aortic wall calcification has been noted in aortic allograft clinical 
explants, ${ }^{19,20}$ and valve leaflet degeneration with and without calcification has also occurred. ${ }^{1-5}$ Furthermore, aortic and pulmonary allograft obstruction due to protruding aortic wall mineral deposits has been described in clinical retrievals of cryopreserved aortic allografts. ${ }^{19,20}$ Aortic wall calcification also has been reported experimentally, and may compromise the use of stentless glutaraldehyde-crosslinked porcine xenograft valves containing lengthy aortic segments.

In addition to the above-described animal models, several groups have studied allograft degeneration using microsurgical aortic valve allograft implants in rats, in which valved aortic segments are inserted as abdominal aortic vascular grafts in recipient rats. ${ }^{36-39}$ The small size of the rat aortic allograft precludes quantitation of the valve cusp calcium concentration, although scanning electron microscopy with energy dispersive X-ray analysis has been used to semiquantitate calcium distribution in aortic valve allograft cusps in rat microsurgical explants. ${ }^{37-39}$ However, no previous investigations have utilized this rat microsurgical aortic model for investigating the effects of glutaraldehyde crosslinking on aortic valve cusps and aortic wall mineralization. A recent study using rat microsurgical aortic allografts has reported inhibition of fresh aortic allograft valve cusp mineralization due to the systemic administration of calcium channel blocker. ${ }^{39}$ Nevertheless, this model has not otherwise been characterized.

Thus, the present study followed the temporal evolution of calcification in this model and tested the following hypotheses: 1) Glutaraldehyde crosslinking potentiates calcification of aortic allograft implants in a rat microsurgical animal model; 2) young age accelerates the progression of calcification in this animal model; and 3) Calcification inhibitors used as either pretreatments or local controlled release polymer coimplants inhibit mineral deposition in this model system.

\section{METHODS}

\section{Materials}

Glutaraldehyde was obtained from Polysciences (Warrington, PA). Ferric chloride and aluminum chloride were obtained from Sigma (St. Louis, MO). Ethanehydroxydiphosphonate (disodium and calcium salts, as well as $\left[{ }^{14} \mathrm{C}\right]$-labeled) was provided by Procter and Gamble (Cincinnati, $\mathrm{OH}$ ). Aminopropanehydroxanediphosphonate (APD) was provided as a gift from Professor Gershon Golomb of the Hebrew University of Jerusalem. The silicone rubber elastomer for polymeric implants used was Silastic Q7-4840 (Dow Corning, Midland, MI).

\section{Allograft harvesting and implant procedures}

Male Brown Norway and Lewis rats were obtained from Charles River Laboratories (Burlington, MA). NIH guidelines for the care and use of laboratory animals (NIH Publication No. 85-23 Rev. 1985) were observed in these investigations. For studies of immature animals, 3-week-old (50 g), male weanling rats from each strain were used. For studies of mature animals, 3-month-old ( $250 \mathrm{~g}$ ), male rats were utilized. Allograft harvesting was carried out by anesthetizing the Brown Norway rats with pentobarbital $(6.5 \mathrm{mg}$ per 100 body wt), followed by heparinization with $100 \mathrm{U}$ of heparin given intravenously. A thoracotomy was then performed, and the heart and ascending aorta were isolated and removed from the animals chest. The basal region of the heart, including the sinus of Valsalva with the adjacent myocardium as well as the ascending aorta, were rapidly removed and perfused with heparinized saline $(10 \mathrm{U} / \mathrm{ml})$ with a peristaltic pump at $2 \mathrm{ml} / \mathrm{min}$ at $25^{\circ} \mathrm{C}$. Fresh aortic allografts were implanted after approximately $30 \mathrm{~min}$ perfusion.

Glutaraldehyde-crosslinked allografts were prepared as follows: After a few minutes of heparinized saline perfusion (as above), allografts were perfused with $0.6 \%$ glutaraldehyde solution in $0.05 \mathrm{M}$ Hepes (pH 7.4) for $1 \mathrm{~h}$ using a peristaltic pump at the same flow rate. Following this, the perfused and fixed allografts were stored in $0.2 \%$ glutaraldehyde, $\mathrm{pH} 7.4$, in the previously described ${ }^{22}$ buffer at room temperature on an orbital shaker at $100 \mathrm{rpm}$ for at least 7 days.

Implantation of the rinsed and either fresh or fixed allografts was carried out by anesthetizing Lewis rats as the recipients using an intramuscular ketamine injection $(60 \mathrm{mg} / \mathrm{kg})$ and intraperitoneal pentobarbitol $(15 \mathrm{mg} / \mathrm{kg}$ ). Valved aortic allografts (either fresh or glutaraldehyde crosslinked) were removed from their storage solutions and rinsed three times with saline, and the right coronary aortic valve cusp was removed, just prior to the anastomotic surgery. A laparotomy was performed, and the abdominal aorta was carefully exposes. A $1.5-\mathrm{cm}$ length of recipient aorta just below the renal arteries was cross clamped at each end, and a cut was made in the recipient aorta equidistant to the two clamps. Allograft implantation was carried out under a 10-power binocular dissecting microscope by interposing the valved allograft with the aortic valve at the proximal end of the anastomosis and the nonvalved portion of the graft at the distal end. Aortic suturing was carried out using 8-0 or 10-0 silk. Following graft interposition, anastomoses were unclamped and checked for leakage. Afterward, the abdominal incision was then closed with mattress sutures, and the animals were recovered. 
No anticoagulation was used during the surgical procedures.

Some of the allografts were implanted with silicone rubber-controlled release drug delivery systems, or vehicle matrices, not containing drugs, wrapped periadventitially around the aorta. Slab matrices, approximately $8 \times 3 \mathrm{~mm}$ (thickness $0.35 \mathrm{~mm}$ ) and formulated as described below, were sutured in place (with 8-0 silk suture) circumferentially surrounding the entire aortic allograft along its entire length. The elastomeric films were wrapped snugly around the aorta, leaving virtually no space between the polymer and the aorta, thereby mediating direct contact.

\section{Controlled release drug delivery systems: formulation and characterization}

EHDP-polymer matrices were formulated using either NaEHDP $(20 \% \mathrm{wt} / \mathrm{wt})$ in silicone rubber or mixtures of the sodium and calcium salts of EHDP in a $1-1$ ratio (15\% and $15 \%)$, dispersing this mixture in a $30 \% \mathrm{wt} / \mathrm{wt}$ loading in Silastic Q7-4840 (components A and $\mathrm{B}$ ), and vulcanizing at $70^{\circ} \mathrm{C}$ temperature for $3 \mathrm{~h}$ until polymerization had occurred as previously described. ${ }^{40}$ Some of the matrices were formulated with $\left[{ }^{14} \mathrm{C}\right]-\mathrm{EHDP}$ (specific activity $58.6 \mathrm{nc}_{\mathrm{i}} / \mathrm{ng}$ ), and these matrices were utilized for the in vitro radioactive release kinetics studies. In vitro assessment of controlled release matrix drug delivery used $\left[{ }^{14} \mathrm{C}\right]$ matrices $(8 \times 3 \mathrm{~mm})$ and $5.0-\mathrm{ml}$ volumes of $\mathrm{pH} 7.4$ Hepes $(0.05 \mathrm{M})$ ) was carried out with periodic sampling over time at $37^{\circ} \mathrm{C}$. The incubation buffer was frequently changed to maintain nearly perfect sink conditions. Drug release kinetic data were plotted as the cumulative percentage of the initially contained drug released over time. ${ }^{40}$

\section{Pretreatment of glutaraldehyde-crosslinked allografts with either $\mathrm{AlCl}_{3}, \mathrm{FeCl}_{3}, \mathrm{APD}$, or sodium dodecyl sulfate (SDS)}

To investigate the calcification inhibition of the above inhibitors, glutaraldehyde crosslinked aortic valve allografts were incubated in aqueous solutions of either $0.1 \mathrm{M} \mathrm{AlCl}_{3}, 0.1 \mathrm{M} \mathrm{FeCl}_{3}$, or $1 \%$ SDS for 24 $\mathrm{h}$ prior to implantation. ${ }^{41,42}$ Other crosslinked al-

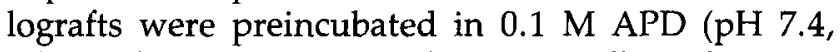
adjusted with $\mathrm{NaOH}$ ). The aortic allografts were rinsed free of pretreatment solutions with copious volumes of normal saline, prior to the allograft implantation.

\section{Explant assessment}

At the time of allograft retrieval, rats were anesthetized with sodium pentobarbitol $(6.5 \mathrm{mg} / 100 \mathrm{~g}$ body $w t$ ), and a laparotomy was performed isolating the abdominal aorta. The allograft was carefully dissected free of connective tissue adhesions, and the animals were then heparinized ( $100 \mathrm{U}$ IV). The allografts were next crossclamped just outside the sites of the anastomoses, and the grafted segment was surgically removed from each subject. At the time of sacrifice, blood via cardiac puncture, and a bone biopsy (femoral head) was obtained from each animal for calcium-phosphorus analyses and morphologic assessment. ${ }^{42}$

Explanted valved aortic allografts were immediately perfused with heparinized saline (10 $\mathrm{U}$ of heparin $/ \mathrm{ml}$ ) for $3 \mathrm{~min}$ at $25^{\circ} \mathrm{C}$ to rinse the grafts free of adherent blood, and then transferred to a solution of Karnovsky's fixative. Following at least $24 \mathrm{~h}$ of immersion, each allograft was dehydrated in graded aqueous ethanol solutions. Specimens were then cut either transversely through the level of the aortic root or bisected longitudinally, embedded in glycolmethacrylate, sectioned at 2-3 $\mu \mathrm{m}$, and stained with hemotoxilin and eosin for overall morphology and von Kossa's reagent (for calcium phosphates).

Samples prepared for assessment of calcification utilized the sinus of Valsalva of the graft, including the valve, and adjacent ascending aorta and sent for morphologic assessment. The remaining aortic segment of the allograft was freeze-dried to a constant weight, and subjected to acid hydrolysis $(6 \mathrm{~N} \mathrm{HCl})$ at $100^{\circ} \mathrm{C}$ as previously describes. ${ }^{42}$ Aliquots of the hydrolyzed samples were analyzed for calcium levels using atomic absorption spectroscopy. Representative femurs from each treatment group were also examined by similar histologic techniques to those described above, to assess growth plate and mineralization morphology. Data were expressed as mean \pm standard error of the mean. Statistical differences between groups were assessed using the Student $t$ test.

\section{RESULTS}

\section{Postsurgical survival and graft patency}

A total of $1.6 \%$ of all animals (four of 251) undergoing the aortic valve allograft procedure died due to the surgery itself. In addition, $1.0 \%$ of the young animals, compared to $4.5 \%$ of the mature animals, died following allograft implantation. Also, whether glutaraldehyde-crosslinked allografts or fresh allografts were used did not seem to influence survival. Of the 70 animals undergoing fresh allograft implantation, two died during the course of the experiment. Of these fresh allograft fatalities, one appeared to be due to ruptured allografts. In addition, two animals with crosslinked aortic allografts died during the course of the study. Two of these fatalities were due to allograft rupture. 

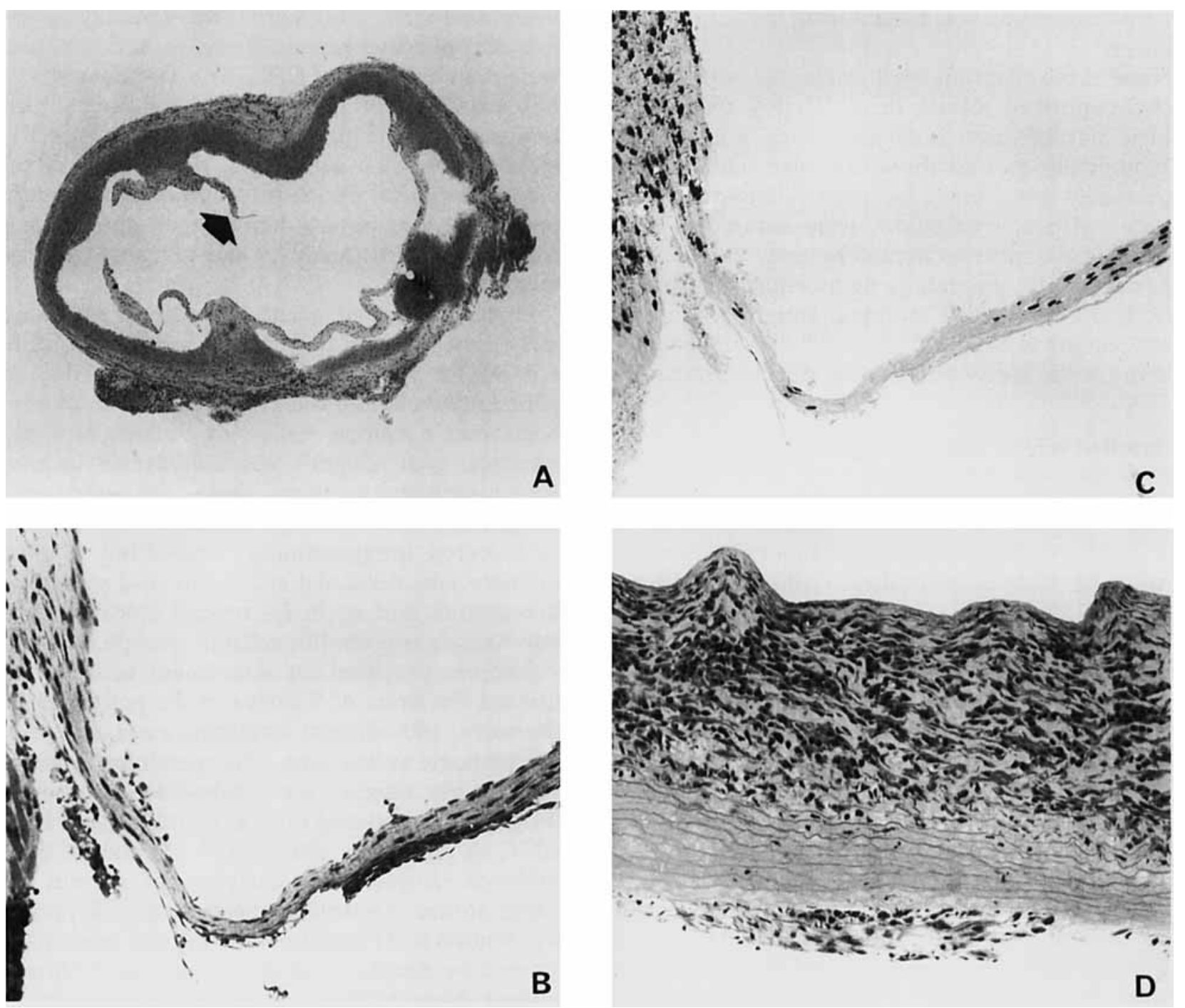

Figure 1. Pathology of explanted rat aortic allografts. (A) Overall anatomy, low power. A cusp is marked by an arrow. (B and C) Glutaraldehyde-fixed cusp implanted 1 week, indicating early gross intrinsic calcific deposits. (d) Fresh aortic wall, implanted 3 weeks. There is extensive mononuclear inflammation, but no calcification was present on corresponding section stained with von Kossa's reagent. (E) Glutaraldehyde-fixed aortic wall, implanted 4 weeks. Extensive calcification is evident. ( $F$ ) Inhibition of aortic wall and cuspal calcification by polymer-based EHDP. (A and B) stained with hematoxylin and eosin; (C-F) stained with von Kossa's reagent (magnification: A, 35×; B, C, E, and F, 140×; D, 175×).

Animals co-implanted with periadventitial polymer films did not have an increased mortality or morbidity due to this procedure. At the time of sacrifice, a dense connective tissue pannus was noted around the allograft-polymer implantation region. The polymer co-implanted allografts, however, were patent in all surviving animals, and did not differ in gross appearance from the allografts retrieved from the nonpolymer studies. Morphologic observations are summarized in Figure 1.

\section{Cuspal observations}

All explanted valves were free of thrombus formation. Valve cusps, whether fresh of glutaraldehydecrosslinked, tended to be overgrown by the fibrous connective tissue arising from the site of the anasto- moses. However, in shorter-term implants, and more commonly in fresh allografts, freely moving aortic valve cusps could often be identified (Fig. 1). Technical difficulties frequently led to loss or obliteration of the cusps during processing of the very small samples for microscopic analysis. Nevertheless, morphologic examinations were possible on some aortic valve cusps. Mineralization was observed in glutaraldehyde-crosslinked cusps, but not in fresh cusps (Fig. 1B-E). Calcific deposits were intrinsically localized (i.e., within the cuspal structure), and were not associated with thrombosis.

\section{Aortic wall calcification}

Aortic wall calcification was grossly apparent, and was associated with progressive stiffness in the 


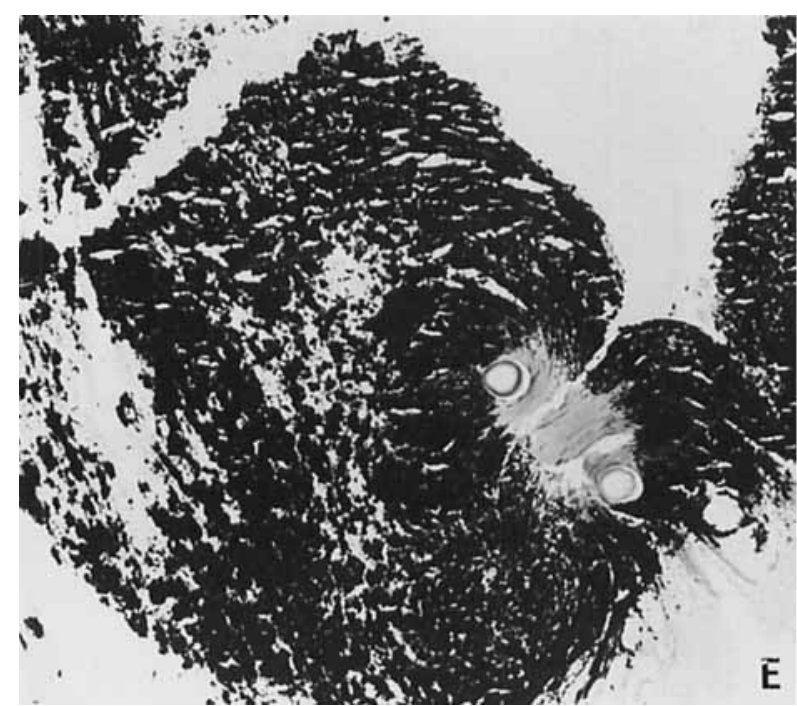

Figure 1.

longer duration implants. Glutaraldehyde-crosslinked aortic wall grafts were more severely calcified than fresh aortic explants (Figs. 1 and 2). Quantitative assessment of aortic wall calcification revealed an ageand time-dependent increase in aortic calcium accumulation, as shown below in Table I and Figure 2. Immature animals demonstrated significantly more aortic wall calcification in the 30-day allograft explants. Calcification by 60 days was equally severe between the two age groups. There were higher levels of calcium accumulated in the crosslinked aortic wall samples than were detected in the noncrosslinked explants. Morphologic assessment of the retrieved calcified aortic segments revealed calcific deposit localization in the media (Fig. 1C and E).

\section{Inhibitor studies: Aortic cusp observations and aortic wall data}

This subset of experiments was carried out using only glutaraldehyde-crosslinked implants. Almost complete inhibition of calcification (Fig. 3) was found with pretreatment of aortic allografts with either ferric chloride $(P<.001)$, or aluminum chloride $(P<$ $.001)$. SDS pretreatment partially inhibited aortic allograft calcification $(P<.001)$, and APD was ineffective. In addition, morphologic assessment revealed no qualitative differences in aortic allograft calcification morphology between the aortic allografts implanted with control (nondrug) periadventitial polymers and those implanted without polymer matrices (data not shown).

Controlled release EHDP formulations (Silastic, Ca, or $\mathrm{Ca}-\mathrm{Na} \mathrm{EHDP}$ ) demonstrated sustained diphosphonate release in vitro (Fig. 4). Adventitial controlled release EHDP matrices (containing a 1-1 mixture of

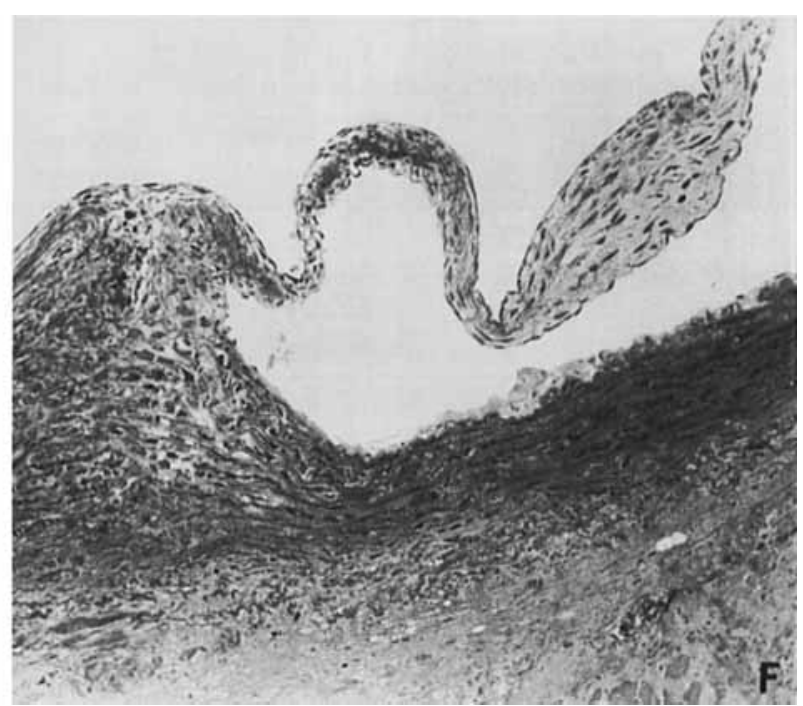

(Continued)

calcium and sodium EHDP), were the most effective of all techniques used (Figs. 1 and 5) for inhibiting glutaraldehyde-crosslinked aortic wall calcification $(P$ $<.001)$. Controlled release Ca EHDP was less effective, and did not differ significantly from control.
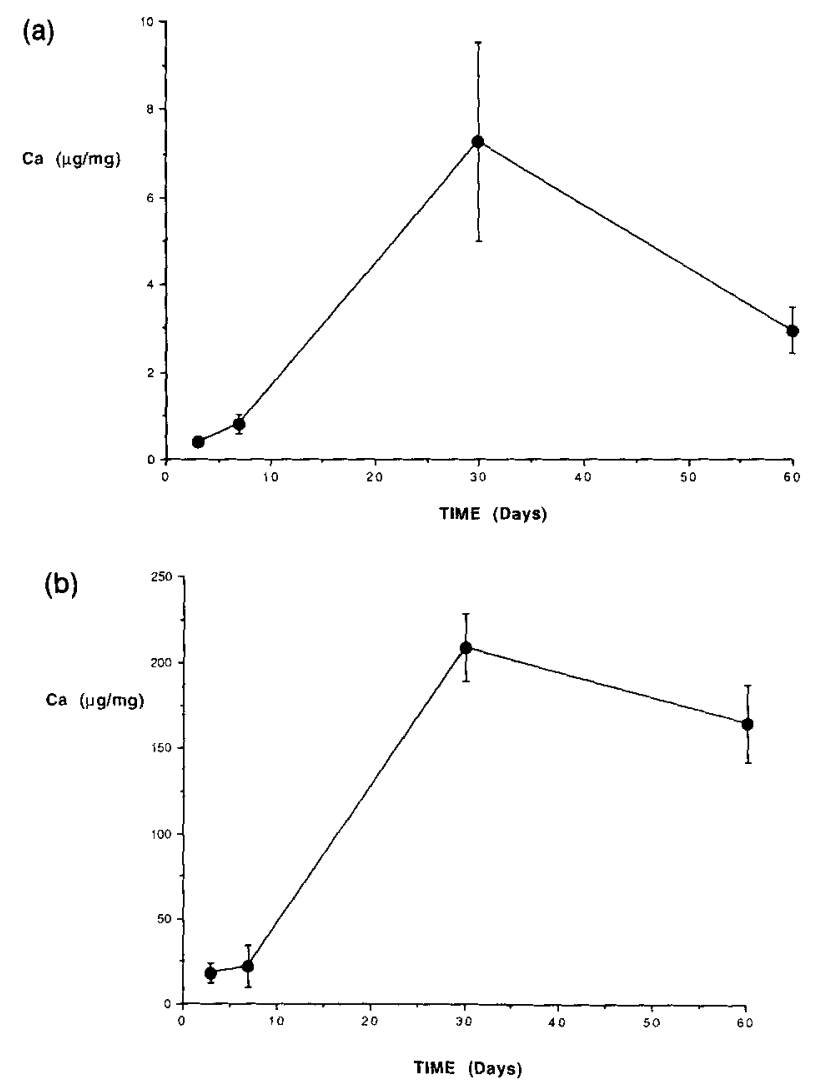

Figure 2. The time course of aortic wall calcification in rat allografts, comparing grafts in the 3-week-old male rat recipients where fresh aortic allografts are demonstrated to accumulate less bulk calcium (a) compared to glutaraldehyde-crosslinked allografts (b), demonstrating severe calcification at very high levels after 30 days. 
TABLE I

The Effect of Age on Calcification of Glutaraldehyde-Crosslinked Aortic Allograft in Rats

\begin{tabular}{lrcr}
\hline $\begin{array}{c}\text { Subjects } \\
\text { (Age) }\end{array}$ & $N$ & $\begin{array}{c}\text { Duration of } \\
\text { Implant }\end{array}$ & \multicolumn{1}{c}{$\begin{array}{c}\text { Calcium } \\
(\mu \mathrm{g} / \mathrm{mg})\end{array}$} \\
\hline Immature (3 wk) & 19 & 30 day & $209.3 \pm 19.9$ \\
Mature (3 mo) & 8 & 30 day & $43.9 \pm 18.9$ \\
Immature & 5 & 60 day & $164.7 \pm 22.9$ \\
Mature & 4 & 60 day & $184.4 \pm 4.24$ \\
\hline
\end{tabular}

No adverse effects of any of the inhibitor pretreatments of controlled release administrations were found to have occurred, as reflected in the normal overall somatic growth and morphology studies, which revealed normal femoral growth plate architecture and mineralization regardless of inhibitor exposure (data not shown).

\section{DISCUSSION}

The present study has characterized a rat microsurgical model of valved aortic allograft mineralization, comparing fresh and glutaraldehyde-crosslinked tissue in both immature and mature subjects. The primary results of the study were that calcification of the allograft aortic wall increased over time, with glutaraldehyde-crosslinked allograft mineralization more severe than that occurring with fresh allografts. In addition, younger animals had more extensive early aortic wall calcification than did mature subjects. Despite this, 60-day explants demonstrated little age difference in allograft aortic wall explant calcification between mature and immature subjects.

However, the assessment of valvular calcification was problematic, since valve cusps were frequently overgrown with fibrous connective tissue, or were

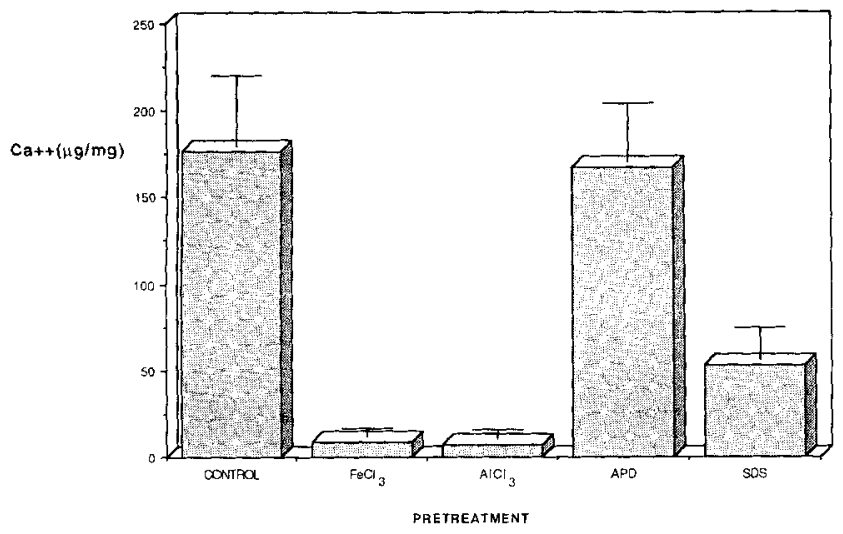

Figure 3. Inhibition of aortic allograft wall calcification (30-day implants) due to 24 -h pre-incubations in various inhibitors as shown: APD, aminopropane-hydroxydiphosphonate; SDS, sodium dodecyl sulfate.
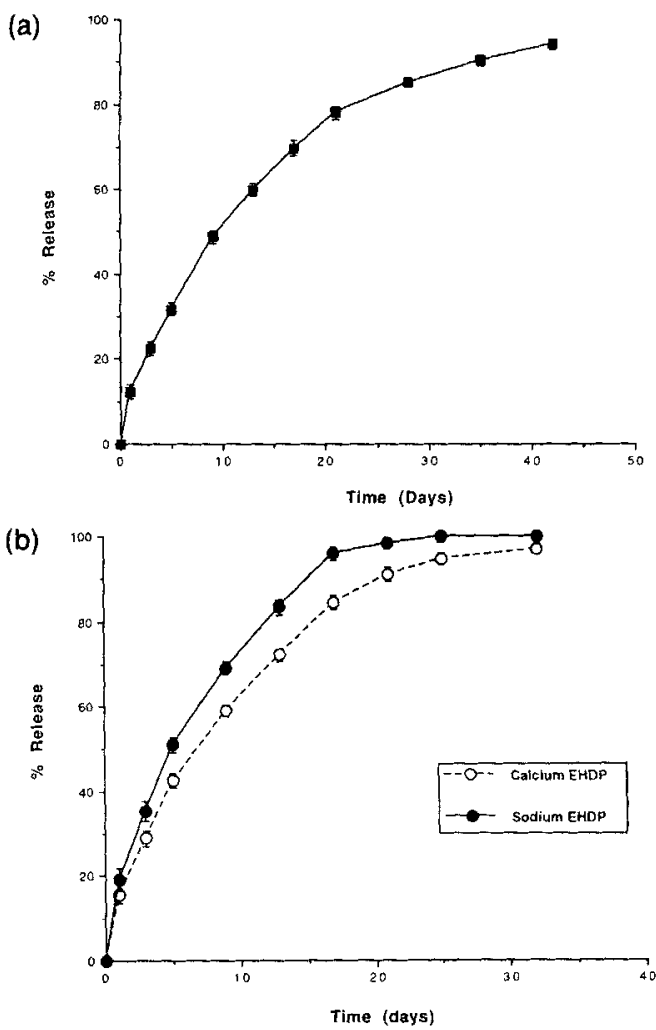

Figure 4. In vitro release of ethanehydroxydiphosphonate (EHDP) from silicone rubber slab matrices as either the calcium salt $(20 \%)$ or equivalently loaded mixtures of sodium and calcium EHDP $(1-1,30 \% \mathrm{wt} / \mathrm{wt})$. As indicated, sustained release of EHDP from both types of matrices was observed over time, with prolongation of release duration due to the sole use of calcium EHDP (a). The calcium fraction (b) also demonstrated sustained release compared to the sodium EHDP coincorporated (b) in other in vitro studies.

lost during processing. Nevertheless, qualitative assessment of cuspal calcification revealed mineral deposits to be most severe in the crosslinked allografts compared to fresh tissue. Aortic wall calcification was consistently observed in both, and appeared to be more severe in explants pretreated with crosslinking agents. Furthermore, anticalcification efficacy was demonstrated with almost complete inhibition of calcification due to $\mathrm{AlCl}_{3}$ or $\mathrm{FeCl}_{3}$ pretreatment, and also for controlled released EHDP. SDS pretreatment partially inhibited aortic allograft calcification, and APD was ineffective

\section{Model characterization}

The results of this study indicate that, while valve cusp mineralization could be produced with this model system, the cusps results tended to be unreliable, since the outcome was often complicated by either fibrous tissue overgrowth, inability to locate 


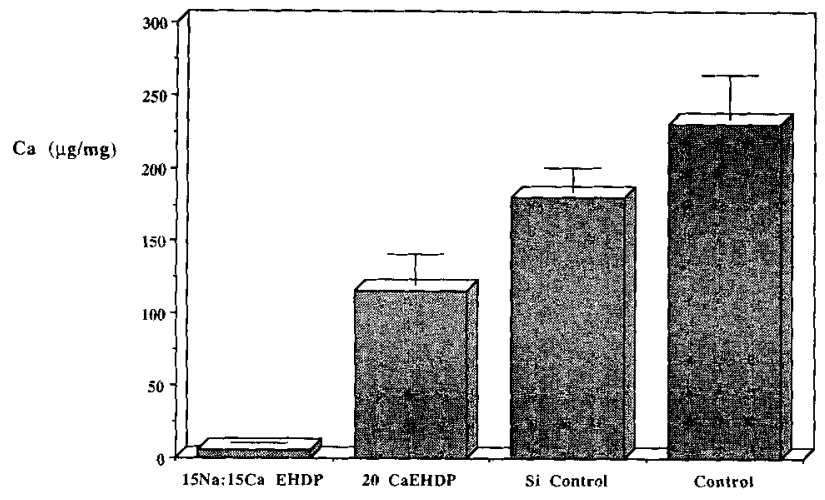

Figure 5. Inhibition of aortic wall allograft calcification with controlled-release polymers compared to both control valved aortic allografts with and without a periadventitial non-drug matrix. All aortic wall specimens were glutaraldehyde crosslinked. As shown, sustained release matrices with either $30 \%$ sodium ethanehydroxydiphosphonate (EHDP) or $15 \% / 15 \%$, sodium/calcium EHDP were most effective. However significant inhibition of calcification was not observed with $20 \%$ calcium EHDP matrices. Allografts coimplanted with nondrug-containing silicone (Si) rubber polymers calcified comparable to control, and statistically did not differ from control.

cusps, or loss during processing. Nevertheless, some useful information was derived from these studies of cuspal mineralization and some possibilities exist for enhancing the valvular calcification outcome in this type of model system.

More useful data were derived, however, from analyses of the aortic wall mineralization. Severe aortic wall calcification was produced by this model, and, as shown in previous studies, ${ }^{22,42}$ was enhanced by the glutaraldehyde-crosslinking techniques used, as well as young age of the recipient subjects. ${ }^{22}$ Investigations of the allograft aortic wall calcification were also revealing in terms of being comparable in many respects to clinicopathologic observations. ${ }^{1-5}$ It is possible that more productive use could be made of studies of calcification in mature rats by extending allograft calcification experiments beyond the 60 days utilized in the present study. However, the calcium levels observed in the present mature rat study were near maximal levels previously observed, ${ }^{42}$ and therefore, the further aggravation of calcific deposits may not provide revealing information.

These aortic wall studies also helped to confirm potent anticalcification strategies, which appeared to be promising in earlier subdermal studies. Similarly, a number of strategies which either appeared to be promising in subdermal studies, such as $\mathrm{APD}^{43}$ or prevented leaflet calcification in the circulation, such as SDS ${ }^{29,30}$ were not useful for preventing aortic wall mineral deposits.

Although our study investigated allograft implants using dysgeneic strains of rats, and made no attempt to sort out possible immune responses, previous studies have shown immune related differences. ${ }^{38}$ An earlier study, examining valve calcification using scanning electron microscopy and energy-dispersive $X$-ray analysis indicated that dysgeneic valve leaflet calcification was more severe than that seen in syngeneic animals with respect to fresh allografts. ${ }^{38}$ However, these investigations did not explore effects in glutaraldehyde pretreated allografts, nor did they investigate aortic wall calcification. Previous results from our group using subdermal implants of porcine aortic leaflets in nude mice compared to normal mice indicated no differences between immune-impaired and normal animals with respect to glutaraldehydecrosslinked heterograft calcification. Further studies should address this specific issue in the animal model described in the present article.

Previously reported investigations with the model reported in this study have focused on fresh aortic allografts, ${ }^{36-40}$ and particularly valve cusp calcification. It is of interest that research by others has not reported the cusp-fibrous tissue overgrowth problem noted in the present investigations, although a recent study of calcium channel blockers inhibiting fresh aortic allograft cusp calcification in rats reported that it was necessary to dissect the cusps after explant to assess calcification. ${ }^{40}$ No prior work by others with a microsurgery animal model has focused on aortic wall calcification. However, aortic wall calcification has been of interest in recent research related to the use of human aortic valve allografts ${ }^{19,20}$ and stentless bioprosthetic valves. ${ }^{14-18}$ Moreover, the aortic wall calcification results were also comparable to those seen in subdermal implant studies of rat aortic allograft calcification. ${ }^{42}$ The aortic allograft calcific deposits also resembled recently reported aortic wall calcifications noted in calcified stentless bioprosthetic heart valves in sheep animal model studies ${ }^{21}$ and in several recent clinical studies reporting human aortic allograft wall mineral deposits. ${ }^{19,20}$

Aortic wall calcification is a potential problem for the long-term use of the stentless bioprosthetic heart valve, since there has been an increasing number of reports of valves aortic allograft (cryopreserved) obstruction due to aortic wall calcific deposits. ${ }^{19,20}$ In particular, recent studies of aortic wall calcification using human allograft valves in children have indicated that it is the most frequent cause of prosthesis failure, and may result in a $50 \%$ or greater failure rate by 3 years postoperation in pediatric populations. ${ }^{19}$

The observed cuspal calcification of the glutaraldehyde-pretreated rat aortic valve demonstrated the intrinsic localization also noted in circulatory explants of porcine aortic bioprosthetic heart valves from both experimental ${ }^{22-28,29-34}$ and clinicopathologic studies. ${ }^{6-10}$ The very small size of the rat aortic valve cusp compared to the larger scale anatomy of the porcine 
aortic cusp makes more direct anatomic comparisons problematic.

\section{Calcification inhibition}

The calcification inhibition results reported in the present study reveal differences between subdermal and circulatory model systems, offering some new strategies for preventing bioprosthetic and allograft aortic wall calcification. For example, in our present circulatory studies, aminopropanehydroxy-diphosphonate (APD) was ineffective when used as a pretreatment for preventing calcification of aortic wall allograft. Previous work by our group, however, demonstrated that APD significantly inhibited the calcification of rat aortic allograft subdermal implants. ${ }^{42}$ Other agents such as $\mathrm{AlCl}_{3}$ or $\mathrm{FeCl}_{3}$ used as allograft pretreatments were successful for inhibiting calcification in both the present circulatory implants and in our previous subdermal studies of the rat aortic allograft model. ${ }^{42}$

Detergent pretreatment has been shown to inhibit bioprosthetic cusp calcification in circulatory ${ }^{29,30}$ and subdermal model studies, ${ }^{\mathbf{4 4 , 4 5}}$ but was incompletely effective for preventing aortic wall calcification in the present study (see SDS results). These data are in agreement with recent results reporting the lack of inhibition of aortic wall calcification using detergent pretreatment with the covalently bound detergent amino oleic acid (AOA) in sheep circulatory studies. ${ }^{21}$

The most profound inhibition of calcification in the present study was observed in animals treated with silicone rubber-controlled release diphosphonate implants. Our studies of periadventitial administration of ethanehydroxy-diphosphonate (EHDP) using silicone rubber matrices demonstrated a virtual complete inhibition of aortic wall and cusp calcification, without any diphosphonate adverse effects. This is in agreement with previous studies of EHDP-controlled release implants by our group, in which controlledrelease EHDP inhibited bioprosthetic cusp calcification, both in subdermal ${ }^{28}$ and sheep circulatory models. ${ }^{46}$ Furthermore, our recent studies have demonstrated metal ion-diphosphonate synergism both in pretreatment protocols and in controlled release polymer co-implant studies of porcine aortic valve cusp mineralization. ${ }^{47}$ Further studies with the present model system might focus on establishing optimal combinations of the most effective inhibitors of aortic wall and aortic cusp mineralization.

Thus, the present study demonstrates that a number of possible therapeutic approaches merit consideration for preventing aortic wall calcification in clinical stentless bioprosthetic heart valve implants. In fact, it may be most beneficial to combine one of the pretreatments or controlled-release implants described in the present studies for inhibiting aortic wall calcification, with one of the better known inhibitors of bioprosthetic cusp calcification, such as detergent pretreatment. This combination of optimal agents could not only result in inhibiting both types (cuspal and aortic wall) of regional calcific deposits observed in bioprosthetic heart valve calcification, but may in fact result in further synergism due to the combined action of the various diverse types of agents.

\section{CONCLUSION}

We conclude that rat aortic allograft calcification using valved allografts anastomosed to the abdominal aorta is a potentially useful model for investigating fresh allograft calcification, as well as the mineralization of glutaraldehyde-crosslinked valved aortic allografts. Valve cusp results, however, may not be comparable to those noted in large animal or human studies, while aortic wall calcification is more comparable. Nearly complete inhibition of the calcification of glutaraldehyde-crosslinked aortic wall allografts was achieved using a number of inhibitors, including controlled-release diphosphonates, and pretreatment with either ferric chloride or aluminum chloride. Aminopropanehydroxydiphosphonate pretreatment was ineffective, and SDS pretreatment was only partially effective for preventing aortic wall calcification in glutaraldehyde-crosslinked allografts.

We thank Mrs. Catherine Wongstrom for her efforts in preparing the manuscript. This work was supported in part by NIH Grant HL 38118, and an American Heart Association grant-in-aid (91-1538).

\section{References}

1. B. B. Barratt-Boyes and A. H. Roche, "A review of aortic valve homografts over a six and one-half year period," Ann. Surg., 170, 483-492 (1969).

2. R. B. Wallace, E. R. Giuliani, and J. L. Titus, "Use of aortic valve homografts for aortic valve replacement," Circulation, 43, 365-373 (1971).

3. O. A. Saravalli, J. Somerville, and K. E. Jefferson, "Calcification of aortic homografts used for reconstruction of the right ventricular outflow tract," $J$. Thorac. Cardiovasc. Surg., 80, 909-920 (1980).

4. D. DiCarlo, M. R. deLeval, and J. Stark, "'Fresh', antibiotic sterilized aortic homografts in extra cardiac valved conduits: Long-term results," Thorac. Cardiovasc. Surg., 32, 10-14 (1984).

5. P. Belcher and D. Ross, "Aortic root replacement-20 years experience of the use of homografts," Thorac. Cardiovasc. Surg., 39, 117-122 (1991).

6. A. Milano, U. Bortolotti, E. Talenti, C. Valfre, E. Arbustini, M. Valente, A. Mazzucco, V. Gallucci, and G. 
Thiene, "Calcific degeneration as the main cause of porcine bioprosthetic valve failure," $\mathrm{Am}$. J. Cardiol., 53, 1066-1070 (1984).

7. S. P. Sanders, R. J. Levy, M. D. Freed, W. I. Norwood, and A. R. Castaneda, "Use of Hancock porcine xenografts in children and adolescents," Am J. Cardiol., 46, 429-438 (1980).

8. C. A. Warnes, M. L. Scott, G. M. Silver, C. W. Smith, V. J. Ferrans, and W. C. Roberts, "Comparison of late degenerative changes in porcine bioprostheses in the mitral and aortic valve position in the same patient," Am. J. Cardiol., 51, 965-968 (1983).

9. S. R. Cohen, M. A. Silver, C. L. McIntosh, and W. C. Roberts, "Comparison of late (62 to 140 months) degenerative changes in simultaneously implanted and explanted porcine (Hancock) bioprostheses in the tricuspid and mitral valve positions in six patients," $\mathrm{Am}$ J. Cardiol., 53, 1599-1602 (1984).

10. F. J. Schoen, J. J. Collins, and L. H. Cohn, "Longterm failure rate and morphologic correlations in porcine bioprosthetic heart valves," Am. I. Cardiol., 51, 957-964 (1983).

11. G. Thiene, U. Bortolotti, M. Valente, A. Milano, F. Calabrese, E. Talenti, A. Mazzucco, and V. Gallucci, "Mode of failure of the Hancock pericardial valve xenograft," Am. J. Cardiol., 63, 129-133 (1989).

12. S. L. Hilbert, V. J. Ferrans, H. A. McAllister, and D. A. Cooley, "Ionescu-Shiley bovine pericardial bioprostheses: Histologic and ultrastructural studies," Am. J. Pathol., 140, 1195-1204 (1992).

13. L. C. Pelletier, M. Carrier, Y. Leclerc, G. Lepage, P. deGuise, and I. Dyrda. "Porcine versus pericardial bioprostheses: A comparison of late results in 1,593 patients." Ann. Thorac. Surg., 47, 352-361 (1989).

14. H. H. Sievers, P. E. Lange, and A. Bernhard, "Implantation of a xenogeneic stentless aortic bioprosthesis: First experience," Thorac. Cardiovasc. Surg., 33, 225-226 (1985).

15. T. E. David, G. C. Ropchan, and J. W. Butany, "Aortic valve replacement with stentless porcine bioprostheses," J. Card. Surg., 3, 501-505 (1988).

16. T. E. David, C. Pollick, and J. Bos, "Aortic valve replacement with stentless porcine aortic bioprosthesis" J. Thorac. Cardiovasc. Surg., 99, 113-118 (1990).

17. M. Hofig, U. Nellessen, M. Mahmoodi, H. H. Sievers, R. Leyh, I. Maurer, A. Bernhard, P. H. Heintzen, and R. Simon, "Performance of a stentless xenograft aortic bioprosthesis up to four years after implantation," $J$. Thorac. Cardiovasc. Surg., 103, 1068-1073 (1992).

18. R. Pallai, D. Spriggings, N. Amarasena, D. J. O'Regan, A. J. Parry, and S. Westaby, "Stentless aortic bioprosthesis? The way forward: Early experience with the Edwards valve," Ann. Thorac. Surg., 56, 88-90 (1993).

19. D. C. Cleveland, W. G. Williams, A. J. Razzouk, G. A. Trusler, I. M. Rebeyka, L. Duffy, Z. Kan, J. G. Coles, and R. M. Freedom, "Failure of cryopreserved homograft valved conduits in the pulmonary circulation," Circulation, 86(Suppl. II), 150-153 (1992).

20. L. Maxwell, J. B. Gavin, and B. G. Barratt-Boyes, "Differences between heart valve allografts an xenografts in the incidence and initiation of dystrophic calcification," Pathology, 21, 5-10 (1989).

21. W. Chen, F. J. Schoen, and R. J. Levy, "Mechanism of efficacy of 2-amino oleic acid for inhibition of calcification of glutaraldehyde pretreated porcine bioprosthetic heart valves," Circulation, 90, 323-329 (1994).
22. R. J. Levy, F. J. Schoen, J. T. Levy, A. C. Nelson, S. L. Howard, and L. J. Oshry, "Biologic determinants of dystrophic calcification and osteocalcin deposition in glutaraldehyde-preserved porcine aortic valve leaflets implanted subcutaneously in rats," $\mathrm{Am}$. J. Pathol., 113, 143-155 (1983).

23. M. C. Fishbein, R. J. Levy, V. J. Ferrans, L. C. Dearden, A. Nashef, A. P. Goodman, and A. Carpentier, "Calcifications of cardiac valve bioprostheses: Biochemical, histologic, and ultrastructural observations in a subcutaneous implantation model system," J. Thorac. Cardiovasc. Surg., 83, 602-609 (1982).

24. F. J. Schoen, H. Harasaki, K. M. Kim, H. C. Anderson, and R. J. Levy, "Biomaterial-associated calcification: Pathology, mechanisms, and strategies for prevention," J. Biomed. Mater. Res., 11-36 (1988).

25. R. J. Levy, F. J. Schoen, W. B. Flowers, and S. T. Staelin, "Initiation of mineralization in bioprosthetic heart valves: studies of alkaline phosphatase activity and its inhibition by $\mathrm{AlCl}^{3}$ or $\mathrm{FeCl}^{3}$ preincubations," $J$. Biomed. Mater. Res., 25, 905-935 (1991).

26. F. J. Schoen, J. L. Kujovich, C. L. Webb, and R. J. Levy, "Chemically determined mineral content of explanted porcine aortic valve bioprostheses: Correlation with radiographic assessment of calcification and clinical data," Circulation, 76, 1061-1016 (1987).

27. D. Hirsch, F. J. Schoen, and R. J. Levy, "Effects of metallic ions and diphosphonates on inhibition of pericardial bioprosthetic tissue calcification and associated alkaline phosphatase activity," Biomaterials, 14, 371-377 (1993).

28. G. Golomb, M. Dixon, M. S. Smith, F. J. Schoen, and R. J. Levy, "Controlled-release drug delivery of diphosphonates to inhibit bioprosthetic heart valve calcification: Release rate modulation with silicone matrices via drug solubility and membrane coating," J. Pharm. Sci., 76, 271-276 (1987).

29. G. R. Barnhart, M. Jones, T. Ishihara, A. M. Chavez, D. M. Rose, and V. J. Ferrans, "Failure of porcine aortic and bovine pericardial prosthetic valves: An experimental investigation in young sheep," Circulation, 66, I150-I153 (1982).

30. E. Arbustini, M. Jones, R. D. Moses, E. E. Eidbo, R. J. Carroll, and V. J. Ferrans, "Modification by the Hancock T6 process of calcification of bioprosthetic cardiac valves implanted in sheep," Am. I. Cardiol., 53, 13881396 (1984)

31. M. Jones, E. E. Eidbo, S. L. Hilbert, V. J. Ferrans, and R. E. Clark, "Anticalcification treatments of bioprosthetic heart valves: In vivo studies in sheep," ]. Card. Surg., 4, 69-73 (1989).

32. G. R. Barnhart, M. Jones, T. Ishihara, A. M. Chavez, D. M. Rose, and V. J. Ferrans, "Bioprosthetic valvular failure: Clinical and pathological observations in an experimental animal model." J. Thorac. Cardiovasc. Surg., 83, 618-631 (1982).

33. H. Harasaki, R. J. Kiraly, G. B. Jacobs, J. L. Snow, and $Y$. Nose, "Bovine aortic and human dura mater valves: A comparative study in artificial hearts in calves," J. Thorac. Cardiovasc. Surg., 79, 125-137 (1980).

34. F. J. Schoen, R. J. Levy, A. C. Nelson, W. F. Bernhard, A. Nashef, and M. Hawley, "Onset and progression of experimental bioprosthetic heart valve calcification," Lab. Invest., 52, 523-532 (1985).

35. F. J. Schoen and R. J. Levy, "Bioprosthetic heart valve failure: Pathology and pathogenesis," Cardiol. Clin., 2, 717-739 (1984).

36. H. el Khatib, S. A. Thompson, and F. M. Lupinetti, 
"Effect of storage at 4 degrees $C$ in a nutrient medium on antigenic properties of rat aortic valve allografts," Ann. Thorac. Surg., 49, 792-796 (1990).

37. J. P. Christy, F. M. Lupinetti, A. H. Mardan, and S. A. Thompson, "Endothelial cell viability in the rat aortic wall," Ann. Thorac. Surg., 51, 204-207 (1991).

38. F. M. Lupinetti, S. Cobb, H. C. Kioschos, S. A. Thompson, K. S. Walters, and K. C. Moore, "Effect of immunological differences on rat aortic valve allograft calcification," J. Card. Surg., 7, 65-70 (1992).

39. H. el Khatib and F. M. Lupinetti, "Antigenicity of fresh and cryopreserved rat valve allografts," Transplantation, 49, 765-767 (1990).

40. S. A. Thompson, G. B. Smith, S. M. Cobb, K. S. Walters, and D. M. Behrendt, "Effects of calcium channel blockers on calcium uptake in rat aortic valve allografts," Circulation, 86, 1973-1976 (1992).

41. C. L. Webb, F. J. Schoen, W. E. Flowers, A. C. Alfrey, C. Horton, and R. J. Levy, "Inhibition of mineralization of glutaraldehyde-pretreated bovine pericardium by $\mathrm{AlCl}$ : Mechanisms and comparisons with $\mathrm{FeCl} 3, \mathrm{LaCl} 3$, and $\mathrm{Ga}(\mathrm{NO} 3) 3$ in rat subdermal model studies," Am. J. Pathol., 138, 971-981 (1991).

42. C. L. Webb, N. M. Nguyen, F. J. Schoen, and R. J. Levy, "Calcification of allograft aortic wall in a rat subdermal model: Pathophysiology and inhibition by $\mathrm{Al}^{+}$and aminodiphosphonate preincubations," Am. J. Pathol., 141, 487-496 (1992).

43. C. L. Webb, J. J. Benedict, F. J. Schoen, J. A. Linden, and R. J. Levy, "Inhibition of bioprosthetic heart valve calcification with aminodiphosphonate covalently bound to residual aldehyde groups," Ann. Thorac. Surg., 46, 309-316 (1988).

44. D. J. Lentz, E. M. Pollock, D. B. Olsen, and E. J. Andrews, "Prevention of intrinsic calcification in porcine and bovine xenograft materials," Trans. Am. Soc. Artif. Intern. Organs, 28, $494-497$ (1982).

45. D. Hirsch, J. Drader, T. J. Thomas, F. J. Schoen, J. T. Levy, and R. J. Levy, "Inhibition of calcification of glutaraldehyde pretreated porcine aortic valve cusps with sodium dodecyl sulfate: Preincubation and controlled release studies," J. Biomed. Mater. Res., 27, 1477-1484 (1993).

46. T. P. Johnston, E. L. Bove, S. F. Bolling, J. A. Boyd, B. L. Ciesliga, G. L. Amidon, F. J. Schoen, and R. J. Levy, "Controlled release of 1-hydroxyethylidene diphosphonate: In vitro assessment and effects on bioprosthetic calcification in sheep tricuspid valve replacement," Int. J. Pharmaceut. 52, 139-148 (1989).

47. D. Hirsch, F. J. Schoen, and R. J. Levy, "Effects of metallic ions and diphosphonates on inhibition of pericardial bioprosthetic tissue calcification and associated alkaline phosphatase activity," Biomaterials, 14, 371-377 (1993).

Received November 15, 1993

Accepted August 5, 1994 